\title{
Perspectives
}

\section{Alberta K-12 ESL Proficiency Benchmarks}

\author{
Kathy Salmon and Mike Ettrich
}

This article provides information on the Alberta K-12 ESL Proficiency Benchmarks with a description of the Benchmarks, their supporting resources, and a commentary on their use in the classroom.

Cet article offre de l'information sur les niveaux de compétence en ALS pour Alberta, de la maternelle à la $12^{e}$ année. Plus précisément, nous décrivons les niveaux de compétence et les ressources sur lesquelles ils s'appuient, et nous offrons des commentaires sur leur emploi en salle de classe.

The Alberta K-12 ESL Proficiency Benchmarks are organized by division: kindergarten, grades 1-3, grades 4-6, grades 7-9, and grades 10-12. They are descriptors of language proficiency in listening, speaking, reading, and writing. The descriptors are arranged in a continuum of seven language competences across five proficiency levels. Several supporting resources have been developed to support classroom teachers in using the Benchmarks, which include:

- illustrative examples,

- writing samples,

- speaking videos,

- tracking sheets,

- division-level summaries.

The Benchmarks assist classroom teachers with establishing baseline proficiency, a focus for language-learning, and ongoing monitoring and reporting. Teachers use them to assess students in the context of the classroom by observing their language use in interactions with peers and teachers, as well as by using writing samples produced in classroom assignments and projects along with formal or informal reading assessments.

Teachers also use the Benchmarks to assess the level of language that students demonstrate in content understanding and then take this information into consideration to set appropriate language-learning expectations and goals.

\section{The Alberta K-12 ESL Proficiency Benchmarks}

In Alberta, the numbers of school-aged children from multilingual families and school-aged newcomers to Canada are increasing. There were 28,837 registered in the Alberta school system in 2003-2004 and 61,499 in 2010-2011 (Alberta Edu- 
cation, 2011). The two urban boards in Calgary have over half of Alberta's English-language learners, with approximately 35,000 students in 2010/2011, and Edmonton's two urban boards have over one quarter, with approximately 17,000 students in 2010/2011. The remaining population of language-learners is located mostly in smaller communities throughout Alberta.

For the four large urban boards in Calgary and Edmonton, English-language learners account for $10-25 \%$ of the student population. In some schools located in culturally diverse neighborhoods, more than $75 \%$ of the students may be learning English as a new language. With a growing population of English-language learners in the province and an urgent need to provide educational supports, Alberta Education commissioned Howard Research \& Management Consulting, Inc. to create A Review of K-12 ESL Education (April 2006), which recommended that English-language proficiency standards be developed in Alberta. A subcommittee from Alberta Education's ESL Advisory Committee reviewed 21 International and Canadian language proficiency standards documents and selected those that best corresponded to the context of learning in Alberta and Alberta Education's programs of studies. Then a provincial committee of 26 Alberta teachers who represented urban and rural school boards used these international documents to develop the framework, language proficiency indicators, and examples for the first draft of the Alberta K-12 ESL Proficiency Benchmarks. This draft was reworked to incorporate elements of the grades 1-9 ESL Benchmarks (2005) created by the Calgary Board of Education. After subsequent revisions, the resulting benchmarks, entitled Alberta K-12 ESL Proficiency Benchmarks, were field-tested in Alberta schools during the 2009-2010 school year. The Benchmarks underwent a final revision based on the results of the field test and are now available to all Alberta teachers of English-language learners at http://www.learnalberta.ca/content/eslapb/index.html.

The primary purpose of the Alberta K-12 ESL Proficiency Benchmarks is to provide descriptions of language proficiency for each grade-level division. They support schools in delivering effective instruction and program-planning for English-language learners by identifying students' initial language proficiency levels, developing consistency in assessment of language proficiency, and promoting collaboration and communication about an Englishlanguage learner's progress among all the student's teachers.

When English-language learners enter the school system, the Benchmarks are used to establish baseline proficiency and to identify the level and types of instructional supports that these learners require to be successful. Each student's current English-language proficiency is assessed on an ongoing basis to monitor growth of language proficiency and to inform instructional planning at each reporting period. In addition, the Benchmarks are used at transition points between grades, schools, and programs to inform programming and instructional decisions. 
The Benchmarks were designed to be used by any teacher of English-language learners including ESL specialists, consultants, and school administrators, as well as K-12 teachers who historically have not seen themselves as ESL teachers, but who are in fact teachers of English-language learners because these students are in their classrooms. The Benchmarks support teachers in assessing, monitoring, tracking, and reporting language proficiency; in communicating with students and parents to develop an understanding of language acquisition; and in planning for explicit language instruction in everyday classroom learning.

\section{Theoretical Framework: From Communicative Competence to Academic Proficiency}

Communicative-language teaching involves developing language proficiency through interactions embedded in meaningful contexts. This approach to teaching provides authentic opportunities for learning that go beyond repetition and memorization of grammatical patterns in isolation. A central concept of the communicative approach to language-teaching is communicative competence, the learner's ability to understand and use language to communicate effectively in authentic (rather than simulated) social and school environments (Canale \& Swain, 1980). The framework is extended to include

Table 1

Communicative Competences

Linguistic Strategic

Understanding and using:

- vocabulary

- language conventions communication (grammar, punctuation and spelling)

- syntax (sentence structure)
Using techniques to:

- overcome language gaps

- plan and assess the effectiveness of

- achieve conversational fluency

- modify text for audience and purpose

Communicative Competence

The ability to understand and use language to communicate effectively in authentic social and school environments.

Sociolinguistic

Having awareness of:

- social rules of language (e.g., formality, politeness, directness)

- nonverbal behaviors

- cultural references (e.g., idioms, expressions, background knowledge)

\section{Discourse}

Understanding how ideas are

connected through:

- patterns of organization

- cohesive and transitional devices 
academic language proficiency (Cummins, 2000), which is the language required of students as they move into more complex, abstract, and cognitively demanding learning tasks.

\section{Alberta K-12 ESL Proficiency Benchmarks: Organized Around the Four Communicative Areas and the Four Language Strands.}

\section{Benchmarks Language Strands}

In each grade-level division, the Benchmarks are organized according to four language strands: listening, speaking, reading, and writing. Based on ageappropriate language development expectations, the kindergarten Benchmarks include only the listening and speaking strands.

\section{Listening}

Listening is the first way for English-language learners to involve themselves in the language-learning process. It involves hearing, processing, and interpreting spoken words by distinguishing sound, rate, pitch, volume, and tone as part of the communication process.

\section{Speaking}

Speaking is a vital component of language-learning that incorporates oral communication elements such as intonation, timing, inflection, speed, rhythm, and pausing, as well as nonverbal elements to support verbal communication such as gesturing and facial expressions.

\section{Reading}

Reading involves decoding (recognizing and understanding letters, numbers, and symbols and how they are used to form words and represent ideas) and comprehension (constructing meaning from words, numbers, and symbols in varied contexts).

\section{Writing}

Writing involves exploring, shaping, and recording one's thoughts and communicating them through various text forms to particular audiences using appropriate tone and voice. Conventions such as spelling, punctuation, and grammar, as well as syntax (sentence structure) and word choice are elements of the writing process.

These strands are viewed as being receptive (receiving information and ideas) and productive (producing information and ideas) or oral (transmitted aloud) and written (transmitted in print). 


\begin{tabular}{lll}
\hline & Receptive & Productive \\
\hline Oral & Listening & Speaking \\
\hline Written & Reading & Writing \\
\hline
\end{tabular}

An English language learner's strengths and areas requiring growth may be focused on receptive or productive strands, and/or oral or written strands. For example, English-language learners may be more (or less) proficient in:

... oral language

\begin{tabular}{lll}
\hline & Receptive & Productive \\
\hline Oral & Listening & Speaking \\
\hline Written & Reading & Writing \\
\hline
\end{tabular}

... written language

\begin{tabular}{lll}
\hline & Receptive & Productive \\
\hline Oral & Listening & Speaking \\
\hline Written & Reading & Writing \\
\hline
\end{tabular}

... receptive language

\begin{tabular}{lll}
\hline & Receptive & Productive \\
\hline Oral & Listening & Speaking \\
\hline Written & Reading & Writing \\
\hline
\end{tabular}

... or productive language

\begin{tabular}{lll}
\hline & Receptive & Productive \\
\hline Oral & Listening & Speaking \\
\hline Written & Reading & Writing \\
\hline
\end{tabular}

\section{Benchmark Competences}

The descriptive indicators in each strand are organized around four communicative areas (competences): linguistic, strategic, sociolinguistic, and discourse. Each of the strands has an additional communicative focus: auditory discrimination, pronunciation, fluency, and editing.

\section{Listening Communicative Focus: Auditory Discrimination}

Auditory discrimination is the ability to hear specific sounds and words and to recognize changes in tone and other nuances of spoken English.

\section{Speaking Communicative Focus: Pronunciation}

Pronunciation involves the ability to produce the sounds and intonations of English effectively so that the speaker is understood. Accents are expected and accepted. 


\begin{tabular}{lllll}
\hline & Listening & Speaking & Reading & Writing \\
\hline Communicative & Linguistic & Linguistic & Linguistic & Linguistic \\
Competences and & Strategic & Strategic & Strategic & Strategic \\
$\begin{array}{l}\text { Strand-specific } \\
\text { Communicative }\end{array}$ & Socio-linguistic & Socio-linguistic & Socio-linguistic & Socio-linguistic \\
Focus & Discourse & Discourse & Discourse & Discourse \\
& & & & \\
& Auditory & Pronunciation & Fluency & Editing \\
& Discrimination & & & \\
\hline
\end{tabular}

Reading Communicative Focus: Fluency

Fluency relates to the rate, ease, and accuracy with which a student decodes and comprehends a text in English.

Writing Communicative Focus: Editing

Editing is the process of reviewing, revising, and refining a text for the purpose of improving it based on English-language conventions (spelling, punctuation, and grammar), word choice, the form of the text, and its intended audience and purpose.

\section{The Benchmarks}

The Alberta K-12 ESL Proficiency Benchmarks have five language proficiency levels in each grade-level division. Each numbered level has a descriptive word label to illustrate what stage the student has reached with respect to language development: 1. Beginning, 2. Developing, 3. Expanding, 4. Bridging, and 5. Extending. The following table shows the complete benchmarks for Grades 4-6 Speaking. 
Table 2

Grades 4-6 Speaking

\begin{tabular}{lllll}
\hline Level 1 & Level 2 & Level 3 & Level 4 & Level 5 \\
Beginning & Developing & Expanding & Bridging & Extending \\
\hline
\end{tabular}

Linguistic: Vocabulary (knowledge of words and their meaning)

Note: The number of words acquired by the end of each level provides educators with an appreciation of vocabulary development from one level to the next. Teachers are neither expected nor encouraged to complete word counts in order to measure language proficiency.

\begin{tabular}{|c|c|c|c|c|}
\hline $\begin{array}{l}\text { Uses a few } \\
\text { words } \\
\text { (approximately } \\
4000 \text { ), } \\
\text { including: } \\
\text { - utility words } \\
\text { - descriptive } \\
\text { words } \\
\text { to express } \\
\text { basic } \\
\text { understanding } \\
\text { or } \\
\text { communicate } \\
\text { immediate } \\
\text { needs and } \\
\text { preferences. }\end{array}$ & $\begin{array}{l}\text { Uses some } \\
\text { words } \\
\text { (approximately } \\
6000 \text { ), } \\
\text { including: } \\
\text { - utility words } \\
\text { - descriptive } \\
\text { words } \\
\text { - subject- } \\
\text { specific words } \\
\text { to convey } \\
\text { understanding } \\
\text { of familiar } \\
\text { topics }\end{array}$ & $\begin{array}{l}\text { Uses more } \\
\text { words } \\
\text { (approximately } \\
7500 \text { ), } \\
\text { including: } \\
\text { - utility words } \\
\text { - descriptive } \\
\text { words } \\
\text { - subject- } \\
\text { specific } \\
\text { words } \\
\text { - academic } \\
\text { words } \\
\text { to convey } \\
\text { understanding } \\
\text { of curricular } \\
\text { concepts. }\end{array}$ & $\begin{array}{l}\text { Uses a range } \\
\text { of words } \\
\text { (approximately } \\
\text { 15,000), } \\
\text { including: } \\
\text { - utility words } \\
\text { - descriptive } \\
\text { words } \\
\text { - subject- } \\
\text { specific } \\
\text { words } \\
\text { - academic } \\
\text { words } \\
\text { to convey } \\
\text { understanding } \\
\text { of curricular } \\
\text { concepts. }\end{array}$ & $\begin{array}{l}\text { Uses a broad } \\
\text { range of words } \\
\text { (approximately } \\
\text { 25,000), } \\
\text { including: } \\
\text { - utility words } \\
\text { - descriptive } \\
\text { words } \\
\text { - subject- } \\
\text { specific } \\
\text { words } \\
\text { - academic } \\
\text { words } \\
\text { to convey } \\
\text { understanding } \\
\text { of abstract } \\
\text { concepts. }\end{array}$ \\
\hline
\end{tabular}

Linguistic: Grammar (ability to form sentences conforming to the rules of English)

\begin{tabular}{|c|c|c|c|c|}
\hline $\begin{array}{l}\text { Uses: } \\
\text { - nouns } \\
\text { simple present } \\
\text { tense } \\
\text { - verbs } \\
\text { - some plurals } \\
\text { with errors and } \\
\text { omissions. }\end{array}$ & $\begin{array}{l}\text { Uses: } \\
\text { - subject } \\
\text { pronouns } \\
\text { - present and } \\
\text { regular past } \\
\text { tense verbs } \\
\text { - plurals } \\
\text { - prepositions } \\
\text { - adjectives } \\
\text { - adverbs } \\
\text { with word- } \\
\text { choice and } \\
\text { word- } \\
\text { placement } \\
\text { errors. }\end{array}$ & $\begin{array}{l}\text { Uses: } \\
\text { - object } \\
\text { pronouns } \\
\text { - irregular verb } \\
\text { tenses } \\
\text { - irregular } \\
\text { plurals } \\
\text { - prepositions } \\
\text { with some } \\
\text { usage errors. }\end{array}$ & $\begin{array}{l}\text { Uses: } \\
\text { - possessive } \\
\text { pronouns } \\
\text { - irregular verb } \\
\text { tenses } \\
\text { - irregular } \\
\text { plurals } \\
\text { - prepositions } \\
\text { - word forms } \\
\text { with occasional } \\
\text { errors. }\end{array}$ & $\begin{array}{l}\text { Uses: } \\
\text { - a variety of } \\
\text { verb tenses } \\
\text { - subject-verb } \\
\text { agreement } \\
\text { - a variety of } \\
\text { word forms } \\
\text { with increasing } \\
\text { accuracy. }\end{array}$ \\
\hline
\end{tabular}


Table 2 (continued)

Grades 4-6 Speaking

\begin{tabular}{|c|c|c|c|c|}
\hline $\begin{array}{l}\text { Level } 1 \\
\text { Beginning }\end{array}$ & $\begin{array}{l}\text { Level } 2 \\
\text { Developing }\end{array}$ & $\begin{array}{l}\text { Level } 3 \\
\text { Expanding }\end{array}$ & $\begin{array}{l}\text { Level } 4 \\
\text { Bridging }\end{array}$ & $\begin{array}{l}\text { Level } 5 \\
\text { Extending }\end{array}$ \\
\hline \multicolumn{5}{|c|}{ Linguistic: Syntax (knowledge of word order and sentence structure) } \\
\hline $\begin{array}{l}\text { Uses: } \\
\text { - familiar } \\
\text { patterned } \\
\text { phrases } \\
\text { - familiar } \\
\text { patterned } \\
\text { sentences. }\end{array}$ & $\begin{array}{l}\text { Forms positive } \\
\text { and negative: } \\
\text { - statements } \\
\text { - questions } \\
\text { - commands. }\end{array}$ & $\begin{array}{l}\text { Adds detail to } \\
\text { positive and } \\
\text { negative: } \\
\text { - statements } \\
\text { - questions } \\
\text { - commands. }\end{array}$ & $\begin{array}{l}\text { Creates longer } \\
\text { detailed } \\
\text { sentences with } \\
\text { clauses. }\end{array}$ & $\begin{array}{l}\text { Varies } \\
\text { sentence } \\
\text { structures. }\end{array}$ \\
\hline
\end{tabular}

Strategic (knowledge of techniques to overcome language gaps)

\begin{tabular}{|c|c|c|c|c|}
\hline $\begin{array}{l}\text { Participates in } \\
\text { familiar } \\
\text { classroom } \\
\text { routines using: } \\
\text { - repeated } \\
\text { words } \\
\text { - familiar } \\
\text { phrases and } \\
\text { questions. }\end{array}$ & $\begin{array}{l}\text { Communicates } \\
\text { with peers and } \\
\text { teachers using: } \\
\text { - known } \\
\text { phrases } \\
\text { - simple } \\
\text { routine } \\
\text { questions. }\end{array}$ & $\begin{array}{l}\text { Interacts using: } \\
\text { - known } \\
\text { expressions } \\
\text { - message } \\
\text { replacement } \\
\text { - questions to } \\
\text { confirm } \\
\text { understanding }\end{array}$ & $\begin{array}{l}\text { Interacts using: } \\
\text { - circumlocution } \\
\text { - personal } \\
\text { connections } \\
\text { - questions to } \\
\text { gather more } \\
\text { information }\end{array}$ & $\begin{array}{l}\text { Initiates and } \\
\text { sustains } \\
\text { communicative } \\
\text { tasks by: } \\
\text { - elaborating } \\
\text { - commenting } \\
\text { - restating } \\
\text { - asking } \\
\text { clarifying } \\
\text { questions. }\end{array}$ \\
\hline
\end{tabular}

Socio-Linguistic (awareness of social and cultural factors influencing the way language is used)

\begin{tabular}{|c|c|c|c|c|}
\hline $\begin{array}{l}\text { Uses familiar: } \\
\text { - expressions } \\
\text { - gestures } \\
\text { to interact in } \\
\text { familiar social } \\
\text { and classroom } \\
\text { contexts. }\end{array}$ & $\begin{array}{l}\text { Uses common: } \\
\text { - expressions } \\
\text { - slang } \\
\text { - idioms } \\
\text { when } \\
\text { interacting with } \\
\text { peers and } \\
\text { adults. }\end{array}$ & $\begin{array}{l}\text { Uses: } \\
\text { - familiar slang } \\
\text { - phrasal verbs } \\
\text { in appropriate } \\
\text { contexts. }\end{array}$ & $\begin{array}{l}\text { Adjusts } \\
\text { speech: } \\
\text { - in formal and } \\
\text { informal } \\
\text { situations } \\
\text { when } \\
\text { interacting with } \\
\text { peers and } \\
\text { adults. }\end{array}$ & $\begin{array}{l}\text { Uses: } \\
\text { - culturally } \\
\text { based idioms } \\
\text { appropriately } \\
\text { when engaging } \\
\text { in a range of } \\
\text { conversational } \\
\text { situations. }\end{array}$ \\
\hline
\end{tabular}


Table 2 (continued)

Grades 4-6 Speaking

\begin{tabular}{|c|c|c|c|c|}
\hline $\begin{array}{l}\text { Level } 1 \\
\text { Beginning }\end{array}$ & $\begin{array}{l}\text { Level } 2 \\
\text { Developing }\end{array}$ & $\begin{array}{l}\text { Level } 3 \\
\text { Expanding }\end{array}$ & $\begin{array}{l}\text { Level } 4 \\
\text { Bridging }\end{array}$ & $\begin{array}{l}\text { Level } 5 \\
\text { Extending }\end{array}$ \\
\hline \multicolumn{5}{|c|}{ Discourse (knowledge of how ideas are organized and connected } \\
\hline $\begin{array}{l}\text { Connects } \\
\text { words in } \\
\text { phrases and/or } \\
\text { short simple } \\
\text { sentences } \\
\text { with: } \\
\text { - "and" and } \\
\text { "then." }\end{array}$ & $\begin{array}{l}\text { Connects } \\
\text { ideas in } \\
\text { sentences } \\
\text { with: } \\
\text { - conjunctions } \\
\text { - time markers } \\
\text { - sequence } \\
\text { markers. }\end{array}$ & $\begin{array}{l}\text { Connects } \\
\text { ideas in related } \\
\text { sentences } \\
\text { using a variety } \\
\text { of: } \\
\text { - conjunctions } \\
\text { - time markers } \\
\text { - sequence } \\
\text { markers. }\end{array}$ & $\begin{array}{l}\text { Connects } \\
\text { ideas in more } \\
\text { complex } \\
\text { sentences } \\
\text { with: } \\
\text { - a variety of } \\
\text { cohesive } \\
\text { devices. }\end{array}$ & $\begin{array}{l}\text { Connects } \\
\text { ideas on the } \\
\text { same topic to } \\
\text { create a logical } \\
\text { flow using: } \\
\text { - transition } \\
\text { words. }\end{array}$ \\
\hline
\end{tabular}

Pronunciation (ability to produce comprehensible speech

\begin{tabular}{|c|c|c|c|c|}
\hline $\begin{array}{l}\text { Imitates some: } \\
\text { - English } \\
\text { sounds } \\
\text { in: } \\
\text { - familiar } \\
\text { routines } \\
\text { although } \\
\text { pronunciation } \\
\text { errors may } \\
\text { interfere with } \\
\text { meaning. }\end{array}$ & $\begin{array}{l}\text { Approximates: } \\
\text { - English } \\
\text { rhythm } \\
\text { - stress } \\
\text { - intonation } \\
\text { in: } \\
\text { - familiar social } \\
\text { and } \\
\text { classroom } \\
\text { routines } \\
\text { although } \\
\text { pronunciation } \\
\text { errors may } \\
\text { interfere with } \\
\text { meaning. }\end{array}$ & $\begin{array}{l}\text { Uses: } \\
\text { - comprehensible } \\
\text { pronunciation } \\
\text { - appropriate } \\
\text { rhythm } \\
\text { - appropriate } \\
\text { intonation } \\
\text { in: } \\
\text { - familiar and } \\
\text { rehearsed } \\
\text { activities } \\
\text { although errors } \\
\text { may still occur. }\end{array}$ & $\begin{array}{l}\text { Uses: } \\
\text { - comprehensible } \\
\text { pronunciation } \\
\text { - appropriate } \\
\text { intonation } \\
\text { in: } \\
\text { - familiar and } \\
\text { rehearsed } \\
\text { activities } \\
\text { with occasional } \\
\text { errors. }\end{array}$ & $\begin{array}{l}\text { Uses: } \\
\text { - comprehensible } \\
\text { pronunciation } \\
\text { - intonation } \\
\text { with increasing } \\
\text { accuracy in: } \\
\text { - unrehearsed } \\
\text { situations } \\
\text { where } \\
\text { dialogue is } \\
\text { spontaneous } \\
\text { (accented } \\
\text { speech is } \\
\text { expected and } \\
\text { accepted). }\end{array}$ \\
\hline
\end{tabular}

\section{Benchmark Levels}

The Alberta K-12 ESL Proficiency Benchmarks reflect how development and academic language expectations increase from one grade-level division to the next. There are five proficiency levels within each division. 


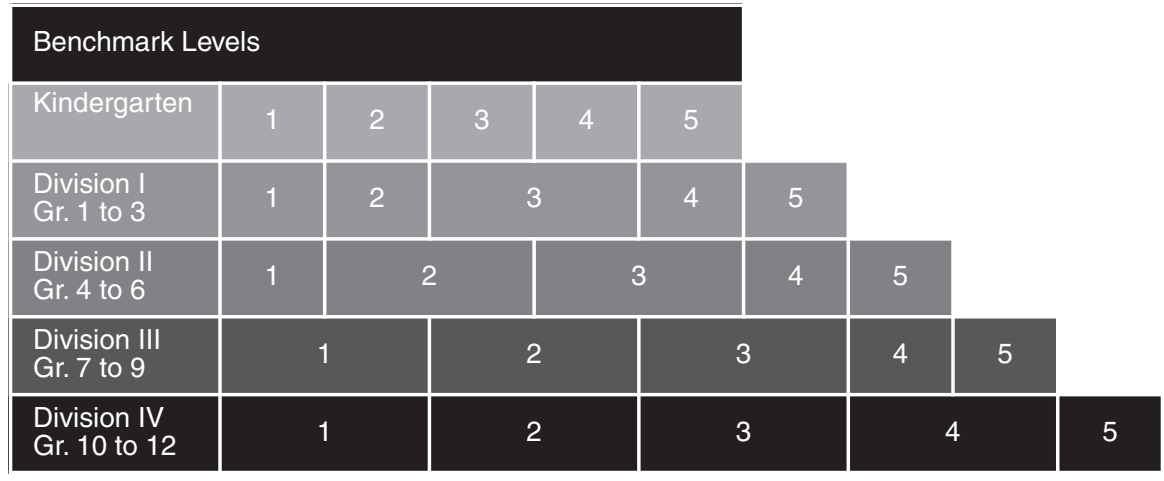

The following tables demonstrate the linguistic syntax competence across the divisions. Notice how the expectations for Level 5 increase as the gradelevel divisions go up.

Table 3

Kindergarten Speaking-Linguistic: Syntax

\begin{tabular}{|c|c|c|c|c|}
\hline $\begin{array}{l}\text { Level } 1 \\
\text { Beginning }\end{array}$ & $\begin{array}{l}\text { Level } 2 \\
\text { Developing }\end{array}$ & $\begin{array}{l}\text { Level } 3 \\
\text { Expanding }\end{array}$ & $\begin{array}{l}\text { Level } 4 \\
\text { Bridging }\end{array}$ & $\begin{array}{l}\text { Level } 5 \\
\text { Extending }\end{array}$ \\
\hline $\begin{array}{l}\text { Forms: } \\
\text { - one- to two- } \\
\text { word } \\
\text { utterances } \\
\text { - memorized } \\
\text { phrases. }\end{array}$ & $\begin{array}{l}\text { Forms: } \\
\text { - familiar } \\
\text { phrases } \\
\text { - simple } \\
\text { patterned } \\
\text { sentences. }\end{array}$ & $\begin{array}{l}\text { Forms: } \\
\text { - simple } \\
\text { sentences } \\
\text { containing: } \\
\text { - subject } \\
\text { - verb } \\
\text { - object. }\end{array}$ & $\begin{array}{l}\text { Manipulates: } \\
\text { - word order } \\
\text { to create } \\
\text { positive and } \\
\text { negative: } \\
\text { - statements } \\
\text { - commands } \\
\text { - questions. }\end{array}$ & $\begin{array}{l}\text { Adds: } \\
\text { - detail } \\
\text { to positive and } \\
\text { negative: } \\
\text { - statements } \\
\text { - commands } \\
\text { - questions. }\end{array}$ \\
\hline
\end{tabular}

Table 4

Grades 1-3 Speaking—Linguistic: Syntax

\begin{tabular}{|c|c|c|c|c|}
\hline $\begin{array}{l}\text { Level } 1 \\
\text { Beginning }\end{array}$ & $\begin{array}{l}\text { Level } 2 \\
\text { Developing }\end{array}$ & $\begin{array}{l}\text { Level } 3 \\
\text { Expanding }\end{array}$ & $\begin{array}{l}\text { Level } 4 \\
\text { Bridging }\end{array}$ & $\begin{array}{l}\text { Level } 5 \\
\text { Extending }\end{array}$ \\
\hline $\begin{array}{l}\text { Uses: } \\
\text { - one or more } \\
\text { words } \\
\text { - memorized } \\
\text { phrases. }\end{array}$ & $\begin{array}{l}\text { Uses: } \\
\text { - simple } \\
\text { sentences. }\end{array}$ & $\begin{array}{l}\text { Uses positive } \\
\text { and negative: } \\
\text { - questions } \\
\text { - statements } \\
\text { - commands. }\end{array}$ & $\begin{array}{l}\text { Adds more } \\
\text { detail to: } \\
\text { - questions } \\
\text { - statements } \\
\text { - commands. }\end{array}$ & $\begin{array}{l}\text { Uses: } \\
\text { - simple } \\
\text { - compound } \\
\text { - complex } \\
\text { sentence } \\
\text { structures. }\end{array}$ \\
\hline
\end{tabular}


Table 5

Grades 4-6 Speaking—Linguistic: Syntax

\begin{tabular}{|c|c|c|c|c|}
\hline $\begin{array}{l}\text { Level } 1 \\
\text { Beginning }\end{array}$ & $\begin{array}{l}\text { Level } 2 \\
\text { Developing }\end{array}$ & $\begin{array}{l}\text { Level } 3 \\
\text { Expanding }\end{array}$ & $\begin{array}{l}\text { Level } 4 \\
\text { Bridging }\end{array}$ & $\begin{array}{l}\text { Level } 5 \\
\text { Extending }\end{array}$ \\
\hline $\begin{array}{l}\text { Uses: } \\
\text { - familiar } \\
\text { patterned } \\
\text { phrases } \\
\text { - familiar } \\
\text { patterned } \\
\text { sentences. }\end{array}$ & $\begin{array}{l}\text { Forms positive } \\
\text { and negative: } \\
\text { - statements } \\
\text { - questions } \\
\text { - commands }\end{array}$ & $\begin{array}{l}\text { Adds detail to } \\
\text { positive and } \\
\text { negative: } \\
\text { - statements } \\
\text { - questions } \\
\text { - commands. }\end{array}$ & $\begin{array}{l}\text { Creates longer } \\
\text { detailed } \\
\text { sentences with } \\
\text { clauses. }\end{array}$ & $\begin{array}{l}\text { Varies } \\
\text { sentence } \\
\text { structures. }\end{array}$ \\
\hline
\end{tabular}

Table 6

Grades 7-9 Speaking—Linguistic: Syntax

\begin{tabular}{|c|c|c|c|c|}
\hline $\begin{array}{l}\text { Level } 1 \\
\text { Beginning }\end{array}$ & $\begin{array}{l}\text { Level } 2 \\
\text { Developing }\end{array}$ & $\begin{array}{l}\text { Level } 3 \\
\text { Expanding }\end{array}$ & $\begin{array}{l}\text { Level } 4 \\
\text { Bridging }\end{array}$ & $\begin{array}{l}\text { Level } 5 \\
\text { Extending }\end{array}$ \\
\hline $\begin{array}{l}\text { Uses: } \\
\text { - two- or three- } \\
\text { word } \\
\text { utterances } \\
\text { - familiar } \\
\text { patterned } \\
\text { phrases } \\
\text { - simple } \\
\text { patterned } \\
\text { sentences } \\
\text { - simple } \\
\text { patterned } \\
\text { questions. }\end{array}$ & $\begin{array}{l}\text { Uses } \\
\text { affirmative and } \\
\text { negative: } \\
\text { - simple } \\
\text { detailed } \\
\text { sentences } \\
\text { - compound } \\
\text { sentences } \\
\text { - questions } \\
\text { - commands. }\end{array}$ & $\begin{array}{l}\text { Uses: } \\
\text { - complex } \\
\text { sentences } \\
\text { - varied word } \\
\text { order. }\end{array}$ & $\begin{array}{l}\text { Adds detail to } \\
\text { a variety of: } \\
\text { - complex } \\
\text { sentences } \\
\text { - compound } \\
\text { sentences. }\end{array}$ & $\begin{array}{l}\text { Uses a variety } \\
\text { of sentence } \\
\text { structures, } \\
\text { including: } \\
\text { - complex } \\
\text { - compound } \\
\text { - conditional. }\end{array}$ \\
\hline
\end{tabular}

\section{Using the Benchmarks}

Teachers can use the Benchmarks in the classroom context. When interacting with student one-on-one or by observing the student working with a partner, small group, or whole class, the teacher observes the listening and speaking competences demonstrated by the student. Reading proficiency assessment requires that the student read a passage of text, retell it, and answer questions informally with the teacher. The teacher may also assess reading proficiency using the Benchmarks following a formal reading, decoding, and comprehension assessment. Writing samples gathered from various subject areas are used to assess a student's written-language proficiency. 
Table 7

Grades 10-12 Speaking—Linguistic: Syntax

\begin{tabular}{|c|c|c|c|c|}
\hline $\begin{array}{l}\text { Level } 1 \\
\text { Beginning }\end{array}$ & $\begin{array}{l}\text { Level } 2 \\
\text { Developing }\end{array}$ & $\begin{array}{l}\text { Level } 3 \\
\text { Expanding }\end{array}$ & $\begin{array}{l}\text { Level } 4 \\
\text { Bridging }\end{array}$ & $\begin{array}{l}\text { Level } 5 \\
\text { Extending }\end{array}$ \\
\hline $\begin{array}{l}\text { Follows: } \\
\text { - patterned } \\
\text { sentences } \\
\text { - phrases } \\
\text { - subject- } \\
\text { verb-object } \\
\text { sentences. }\end{array}$ & $\begin{array}{l}\text { Uses patterned } \\
\text { and predictable } \\
\text { affirmative and } \\
\text { negative: } \\
\text { - statements } \\
\text { - questions } \\
\text { - commands. }\end{array}$ & $\begin{array}{l}\text { Adds detail to } \\
\text { affirmative and } \\
\text { negative: } \\
\text { - statements } \\
\text { - questions } \\
\text { - commands. }\end{array}$ & $\begin{array}{l}\text { Uses: } \\
\text { - compound } \\
\text { sentence } \\
\text { structures } \\
\text { - complex } \\
\text { sentence } \\
\text { structures } \\
\text { - conditional } \\
\text { sentence } \\
\text { structures. }\end{array}$ & $\begin{array}{l}\text { Manipulates } \\
\text { word order to } \\
\text { convey precise } \\
\text { meaning in: } \\
\text { - complex } \\
\text { sentence } \\
\text { structures }\end{array}$ \\
\hline
\end{tabular}

The following excerpt from a tracking sheet shows the teacher's observations of the student's language proficiency level. This excerpt shows Levels 2-4 only. The student was observed in October consistently using the words and and then to connect ideas when writing. The tracking sheet was dated March 2012 to show when the student demonstrated this competence in his or her writing. The teacher wrote observations in Level 3 to capture what the student was working on in Level 3 Discourse.

Table 8

Grades 1-3 Writing: Discourse

\begin{tabular}{|c|c|c|c|c|c|}
\hline $\begin{array}{l}\text { Level } 2 \\
\text { Developing }\end{array}$ & Date & $\begin{array}{l}\text { Level } 3 \\
\text { Expanding }\end{array}$ & Date & $\begin{array}{l}\text { Level } 4 \\
\text { Bridging }\end{array}$ & Date \\
\hline $\begin{array}{l}\text { Connects words } \\
\text { in simple } \\
\text { sentences with: } \\
\text { - "and" or "then" }\end{array}$ & $\begin{array}{l}\text { March } \\
2012\end{array}$ & $\begin{array}{l}\text { Connects ideas } \\
\text { in short, related } \\
\text { sentences with: } \\
\text { • conjunctions } \\
\text { - time markers } \\
\text { Using "so" and } \\
\text { "or" in } \\
\text { sentences. } \\
\text { Beginning to } \\
\text { use "today" to } \\
\text { start sentences } \\
\text { (June 2012). }\end{array}$ & & $\begin{array}{l}\text { Connects ideas } \\
\text { in sentences } \\
\text { using a range } \\
\text { of: } \\
\text { - conjunctions } \\
\text { - time markers } \\
\text { - sequence } \\
\text { markers. }\end{array}$ & \\
\hline
\end{tabular}


Teachers use the tracking sheets to document students' language proficiency levels when communicating with students, parents, and other teachers. Comments about students' language proficiency and goals may be created using the Benchmark indicators.

\section{Example of a Report Card Comment}

In her writing, Soyoung connects sentences with and and then. Sometimes, she uses so and or to connect ideas. To improve her writing, she can begin her sentences with more time markers such as Yesterday, Last night, or On Monday.

\section{Benchmarks Online}

The Alberta K-12 ESL Proficiency Benchmarks are an interactive online digital resource located at www.learnalberta.ca, keyword ESL or via the direct link http://www.learnalberta.ca/content/eslapb/index.html.

The digital format allows teachers to search the Benchmarks by gradelevel division, strand, or competence to isolate particular competences or compare competences. The following chart shows the comparisons for sociolinguistic competences in reading across grades 7-9 and 10-12.

\section{Benchmark Examples}

Illustrative examples are included in the online digital resource. These give sample words, grammar forms, sentence structures, and other information about language proficiency associated with each Benchmark descriptor. Used in conjunction with the Benchmark descriptors, these examples assist teachers in determining their students' language proficiency. Examples often include a quotation of what the student may say, comprehend, or write. The examples encompass various subject or content areas. Those for a particular

\section{READING (GRADES 7-9)}

\begin{tabular}{|c|c|c|c|c|}
\hline Level 1: Beginning & Level 2: Developing & Level 3: Expanding & Level 4: Bridging & Level 5: Extending \\
\hline $\begin{array}{l}\text { Understands: } \\
\text { - the literal meaning } \\
\text { of simple texts on familiar topics. } \\
\text { More info }\end{array}$ & $\begin{array}{l}\text { Understands: } \\
\text { - purposes of a variety of } \\
\text { genres. } \\
\text { More info » }\end{array}$ & $\begin{array}{l}\text { Understands: } \\
\text { - common social expressions } \\
\text { - figurative language } \\
\text { in texts on familiar topics. } \\
\text { More info \# }\end{array}$ & $\begin{array}{l}\text { Understands: } \\
\text { - explicit social references } \\
\text { - explicit cultural references } \\
\text { in a variety of texts. } \\
\text { More info » }\end{array}$ & $\begin{array}{l}\text { Understands implied meaning of } \\
\text { - social references } \\
\text { - cultural references } \\
\text { in context. } \\
\text { More info » }\end{array}$ \\
\hline
\end{tabular}

READING (GRADES 10-12)

\begin{tabular}{|c|c|c|c|c|}
\hline Level 1: Beginning & Level 2: Developing & Level 3: Expanding & Level 4: Bridging & Level 5: Extending \\
\hline $\begin{array}{l}\text { Understands: } \\
\text { - the literal meaning } \\
\text { of simple texts on familiar topics. } \\
\text { More info }\end{array}$ & $\begin{array}{l}\text { Understands: } \\
\text { - common social expressions } \\
\text { - figurative language } \\
\text { in texts on familiar topics. } \\
\text { More info \# }\end{array}$ & $\begin{array}{l}\text { Understands: } \\
\text { - explicit social references } \\
\text { - explicit cultural references } \\
\text { - figurative language } \\
\text { in a variety of texts. } \\
\text { More info » }\end{array}$ & $\begin{array}{l}\text { Understands implied meaning of: } \\
\text { - social references } \\
\text { - cultural references } \\
\text { - figurative language. } \\
\text { in context. } \\
\text { More info » }\end{array}$ & $\begin{array}{l}\text { Understands: } \\
\text { - most cultural references } \\
\text { with or without context. } \\
\text { More info » }\end{array}$ \\
\hline
\end{tabular}




\begin{tabular}{|c|c|c|c|c|}
\hline Level 1: Beginning & Level 2: Developing & Level 3: Expanding & Level 4: Bridging & Level 5: Extending \\
\hline $\begin{array}{l}\text { Participates in familiar classroom } \\
\text { routines using: } \\
\text { - repeated words } \\
\text { - familiar phrases and } \\
\text { questions. } \\
\text { \& Less info } \\
\text { Student uses: } \\
\text { - familiar phrases, such as } 9 \\
\text { need pencil." } \\
\text { - familiar questions, such as } \\
\text { "You my partner?" or "You } \\
\text { help me?" }\end{array}$ & $\begin{array}{l}\text { Communicates with peers and } \\
\text { teachers using: } \\
\text { : known phrases } \\
\text { - simple routine questions. } \\
\text { "Less info } \\
\text { Student uses: } \\
\text { - known phrases, such as } \\
\text { "How was weekend?" } \\
\text { - simple routine questions, } \\
\text { such as "What I do?" (which } \\
\text { could mean "What do I do } \\
\text { next?" or "What should I be } \\
\text { doing?" or "What did I do } \\
\text { wrong?") }\end{array}$ & $\begin{array}{l}\text { Interacts using: } \\
\text { - known expressions } \\
\text {. message replacement } \\
\text { questions to confirm } \\
\text { understanding. } \\
\text { "Less info } \\
\text { Student overcomes language } \\
\text { gaps by using: } \\
\text { - known expressions, such } \\
\text { as " I need help," or " I don't } \\
\text { get it" } \\
\text { - message replacement, } \\
\text { such as when it is too } \\
\text { complicated to explain why } \\
\text { the paper plane won't fly, the } \\
\text { student says, "It doesn't } \\
\text { work: } \\
\text { - questions to confirm } \\
\text { understanding, such as } \\
\text { "What does this mean?" or } \\
\text { "Can you explain it again, } \\
\text { please?" }\end{array}$ & $\begin{array}{l}\text { Interacts using: } \\
\text { - circumlocution } \\
\text {. personal connections } \\
\text { questions to gather more } \\
\text { information. } \\
\text { \& Less info } \\
\text { Student overcomes language } \\
\text { gaps by. } \\
\text { - using circumlocution, such } \\
\text { as when the student doesn't } \\
\text { have the word 'time } \\
\text { capsule," he or she } \\
\text { describes it as, "The thing } \\
\text { like a box where you put } \\
\text { things in and then people } \\
\text { find it later." } \\
\text { making a personal } \\
\text { connection, such as when } \\
\text { the student shares stories } \\
\text { and ideas related to the } \\
\text { topic of discussion } \\
\text {. using questions to gather } \\
\text { more information, such as } \\
\text { "Why ..?", "When...?". } \\
\text { "Where ...?" }\end{array}$ & $\begin{array}{l}\text { Initiates and sustains } \\
\text { communicative tasks by: } \\
\text { - elaborating } \\
\text {. commenting } \\
\text { - restating } \\
\text { - asking clarifying questions. } \\
\text { Sess info } \\
\text { Student may have a } \\
\text { conversation with a peer about a } \\
\text { trip to the aerospace museum, } \\
\text { using strategies such as: } \\
\text { - elaborating, such as "1 really } \\
\text { like planes and especially } \\
\text { the planes used in the wars. } \\
\text { Once I got to sit in the } \\
\text { cockpit of a fighter jet." } \\
\text { - commenting, such as "Did } \\
\text { you see the helicopter? It } \\
\text { was really cool." } \\
\text { - restating, such as "When } \\
\text { you said you went to the } \\
\text { museum, you said that you } \\
\text { thought the veteran that gave } \\
\text { the tour was really } \\
\text { interesting." } \\
\text { - asking clarifying questions, } \\
\text { such as "Did he say his } \\
\text { secret missions to drop } \\
\text { paratroopers and supplies } \\
\text { behind enemy lines?" }\end{array}$ \\
\hline
\end{tabular}

competence typically deal with the same subject area for all five levels to demonstrate what a student may do at various levels of proficiency in a particular classroom context. Varied subject areas and topics are used for the examples to assist teachers in social studies, math, science, and language arts to observe English-language learners' proficiency in the classroom context. The above table shows the Benchmarks descriptors for listening: strategic questioning and the corresponding examples.

The Benchmark examples appear beneath each of the descriptors on a shaded background.

\section{Benchmark Videos}

To support the Benchmarks for speaking, videotapes were created to show students at various proficiency levels engaged with curriculum topics in the classroom. The videos consist of an interview with each student responding to the same questions about the lesson. Then the student and teachers discuss the students' responses in the interview and in the classroom. The teachers assess students' language use to determine their proficiency on the Benchmark competences.

The following excerpts are from a grades 10-12 science lesson on the geological time scale. Students were asked questions about superposition and radiometric dating.

Level 1

Teacher: "What is superposition?"

Student: "Is like super and position, is means over and place." 
Teacher: "What is radiometric dating?"

Student: "Looking on the chemical composition the rock."

Level 2

Teacher: "What is the difference between superposition and radiometric dating?"

Student: "Superposition is used ... um ... I think ... is it physical method? I think so. Cause like the super means over and position means place, and it's like a physical method. And the radiometric dating is a chemical method. We find out absolute age."

Level 3

Teacher: "If you were a scientist and you had a mammoth tusk and a fossil fish, how would you decide which was older than the other?"

Student: "We can see the superposition. The fossils in the superposition. We know that the superposition on the top is the youngest and in the bottom is the oldest. And we can find which date in the layer. The lower layer is older than the top layer."

Teacher: "Now what about radiometric dating?"

Student: "It shows us to find the current of age for the particular layers of the rocks and we can use that to find the age of the fossils."

Level 4

Teacher: "How can you tell which one is older?"

Student: "With superposition, super means over and position means place and so you can determine how old a fossil is. The trilobite is younger than the ammonite by 20 million years. Cause the ammonite is older than the trilobite and the oldest is at the bottom."

Teacher: "What is radiometric dating?"

Student: "It is ... um ... they analyze chemical changes that have occurred to the layer over time so that they can determine the age of the fossil."

Level 5

Teacher: "How would a scientist know that it was over 400 million years old?"

Student: "They would use superposition as well as the radiometric dating. Super means over and position means places so they would look at a rock and see how big it is. And on the top it 
would be the youngest. On the top of the rock level it would be the youngest. And on the bottom would be the oldest. So they would find the oldest soils would be at the bottom and youngest on the top."

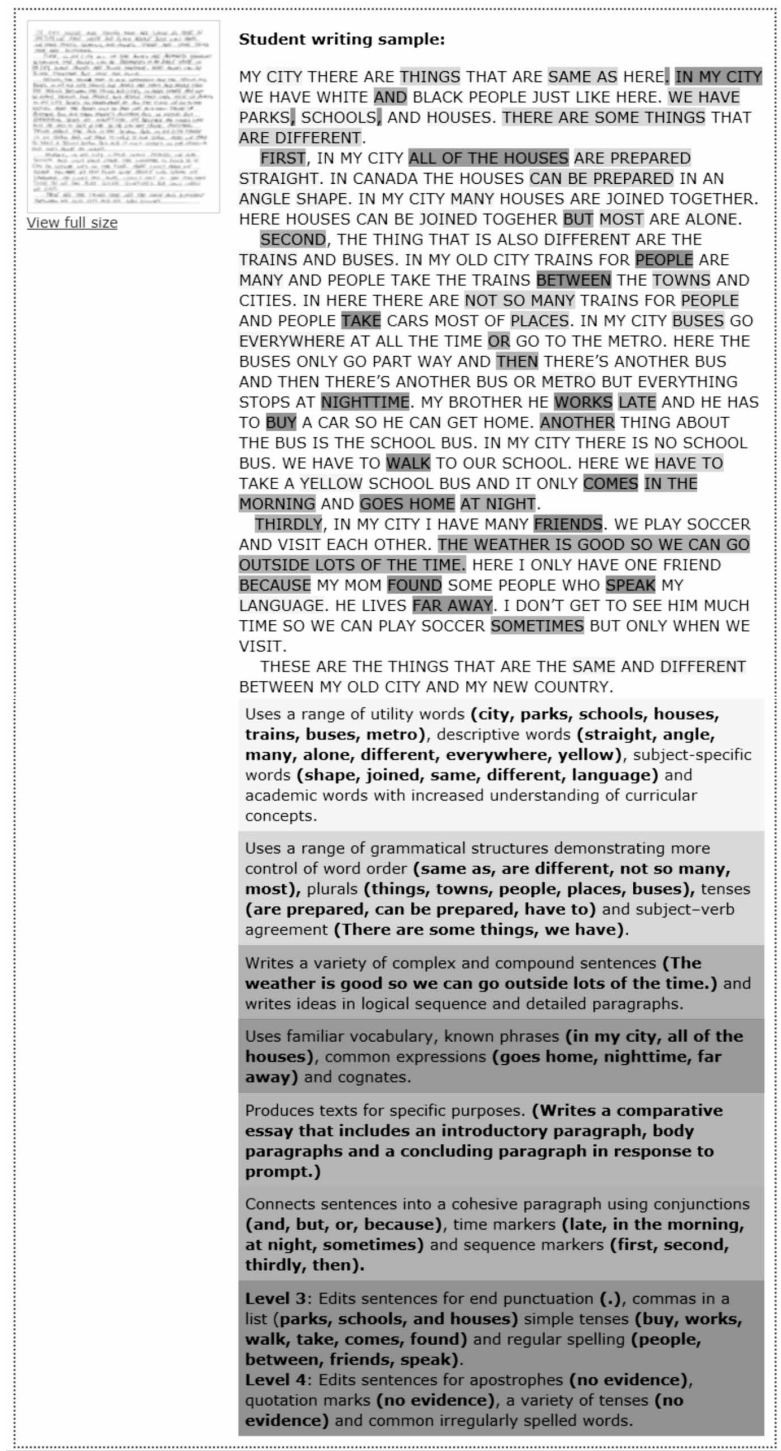

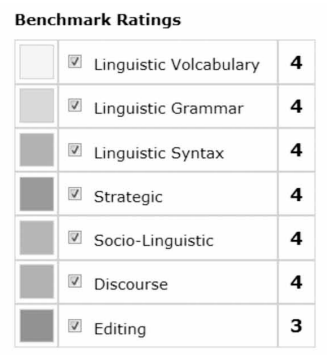

Overall Benchmark Level:

\section{Grade 6: Level 4 Writing Sample}


Teacher: "What is radiometric dating?"

Student: "It determines how old the rock is by looking at the chemicals in the

rock."

Teacher: "What is the difference between superposition and radiometric dating?"

Student: "Superposition is the position of the fossil in the rock so the top or the bottom or the middle. And the radiometric dating is how old the rock actually is."

\section{Writing Samples}

Samples of students' writing for each grade for each of the five levels with comments and benchmark levels assist teachers in determining proficiency levels.

Table 9

Grades 7-9 Speaking

\begin{tabular}{|c|c|c|c|c|}
\hline $\begin{array}{l}\text { Level } 1 \\
\text { Beginning }\end{array}$ & $\begin{array}{l}\text { Level } 2 \\
\text { Developing }\end{array}$ & $\begin{array}{l}\text { Level } 3 \\
\text { Expanding }\end{array}$ & $\begin{array}{l}\text { Level } 4 \\
\text { Bridging }\end{array}$ & $\begin{array}{l}\text { Level } 5 \\
\text { Extending }\end{array}$ \\
\hline $\begin{array}{l}\text { Expresses } \\
\text { basic needs. } \\
\text { Uses: } \\
\text { - familiar words } \\
\text { - words } \\
\text { learned in } \\
\text { class } \\
\text { - phrases } \\
\text { - simple } \\
\text { sentences } \\
\text { - familiar } \\
\text { greetings and } \\
\text { expressions. } \\
\text { Speaks with } \\
\text { some errors } \\
\text { and omissions. }\end{array}$ & $\begin{array}{l}\text { Expresses } \\
\text { needs, } \\
\text { feelings, and } \\
\text { preferences } \\
\text { and responds } \\
\text { to questions. } \\
\text { Uses: } \\
\text { - more utility, } \\
\text { descriptive } \\
\text { and subject- } \\
\text { specific } \\
\text { words } \\
\text { - simple } \\
\text { detailed } \\
\text { sentences } \\
\text { - awareness of } \\
\text { informal and } \\
\text { formal } \\
\text { register. } \\
\text { Speaks with } \\
\text { some } \\
\text { overgeneralizat } \\
\text { ion errors, } \\
\text { such as } \\
\text { misuse of -s } \\
\text { and -ed }\end{array}$ & $\begin{array}{l}\text { Expresses } \\
\text { ideas, makes } \\
\text { statements, } \\
\text { and asks and } \\
\text { answers } \\
\text { questions. } \\
\text { Uses: } \\
\text { - a range of } \\
\text { utility, } \\
\text { descriptive, } \\
\text { subject- } \\
\text { specific and } \\
\text { academic } \\
\text { words } \\
\text { - varied word } \\
\text { order in } \\
\text { complex } \\
\text { sentences } \\
\text { - slang and } \\
\text { idioms. } \\
\text { Speaks with } \\
\text { some subject- } \\
\text { verb } \\
\text { agreement } \\
\text { errors and } \\
\text { tense errors. }\end{array}$ & $\begin{array}{l}\text { Communicates } \\
\text { by clarifying, } \\
\text { commenting, } \\
\text { stating } \\
\text { opinions, and } \\
\text { expressing } \\
\text { agreement and } \\
\text { disagreement. } \\
\text { Uses: } \\
\text { - a greater } \\
\text { range of } \\
\text { vocabulary } \\
\text { - complex, } \\
\text { detailed } \\
\text { sentences } \\
\text { - social } \\
\text { references, } \\
\text { expressions } \\
\text { and idioms in } \\
\text { appropriate } \\
\text { contexts. } \\
\text { Speaks with } \\
\text { some usage } \\
\text { errors. }\end{array}$ & $\begin{array}{l}\text { Communicates } \\
\text { through } \\
\text { discussion, } \\
\text { inquiry, and } \\
\text { persuasion. } \\
\text { Uses: } \\
\text { - a broad } \\
\text { range of } \\
\text { vocabulary } \\
\text { - a variety of } \\
\text { sentence } \\
\text { structures } \\
\text { - humor and } \\
\text { sarcasm in } \\
\text { appropriate } \\
\text { contexts. } \\
\text { Speaks with } \\
\text { occasional }\end{array}$ \\
\hline
\end{tabular}




\section{Division-Level Summaries}

With four strands and seven competences per strand, the Benchmarks consist of 28 rows of information for teachers. Division-level summaries were created to provide a higher-level overview of the information contained in the Benchmarks to serve as a useful introduction to the Benchmarks for teachers who are exploring them for the first time.

On the previous page is a sample of a Division-Level Summary for Speaking in grades 7-9.

\section{Conclusion}

The Alberta K-12 ESL Proficiency Benchmarks are a criterion-referenced assessment to support teachers in assessing English-language proficiency in the context of the classroom. Teachers plan for instruction by using the Benchmark descriptors to embed language instruction in content instruction. They use the Benchmarks to provide relevant information to colleagues for students' transitions and course selection. In addition, the Benchmarks facilitate communication about language proficiency and language acquisition between teachers and students and their parents or guardians. In time, the use of the Benchmarks will lead to increased consistency in language proficiency assessment in Alberta.

\section{The Authors}

Kathy Salmon enjoys supporting teachers of English-language learners in providing explicit language instruction in meaningful and engaging contexts. Most recently, Kathy was Curriculum Manager, ESL for Alberta Education in a three-year secondment from the Calgary Board of Education where she now works as an assistant principal. She has worked as an ESL designate teacher, a learning leader, an ESL specialist and a facilitator for workshops across Alberta. She has her obtained her Bachelor of Education and MEd TESL from the University of Calgary.

Mike Ettrich is the Team Leader for English as a Second Language at Alberta Education. His role is to help provide resources and tools to support teachers of English-language learners and to facilitate collaborative opportunities that build teachers' capacity and enhance the achievement of K-12 English-language learners. Mike's background includes language acquisition pedagogy; educational technology; development of digital, print and video-based learning and teaching resources; and distance learning.

\section{References}

Alberta Education. (2011). ESL enrolment data. Edmonton, AB: School Finance, Alberta Education.

Calgary Board of Education. (2005). English as a second language: English language proficiency benchmarks grades $K$ to nine (3rd ed.). Calgary, AB: Author.

Canale, M., \& Swain, M. (1980). Theoretical bases of communicative approaches to secondlanguage teaching and testing. Applied Linguistics, 1(1), 1-47.

Cummins, J. (2000). Language, power and pedagogy: Bilingual children caught in the crossfire. Clevedon, UK: Multilingual Matters.

Howard Research \& Management Consulting, Inc. (2009). Kindergarten to grade 12 English as a second language literature review update. Edmonton, AB: Alberta Education. 